\title{
Place de l'hydroélectricité dans le bilan énergétique mondial
}

\author{
Share of hydro-electric power \\ in the world energy balance
}

\author{
J. Cotillon \\ Secrétaire Général \\ Commission Internationale des Grands Barrages \\ Contrôleur Général adjt. \\ Direction de l'Equipement - Electricité de France
}

\section{Introduction}

Quelle est la place actuelle de l'énergie hydraulique parmi les autres énergies? Quel est son avenir, sa place dans le bilan énergétique mondial de l'an 2000 par exemple?

1. C'est une énergie renouvelable et cela renseigne déjà sur ses possibilités : comme la plupart de ces énergies, elle est liée aux éléments non transportables d'un site, doit être produite sur place, son transport ne pouvant s'effectuer alors que sous forme d'électricité. L'énergie hydraulique trouve donc l'essentiel de ses applications dans l'électricité.

Les énergies non-renouvelables au contraire sont disponibles sous forme de gisements solides, liquides ou gazeux : leur matière première est plus facilement transportable et concentrable que l'électricitć (l'Australie peut envoyer en Europe son charbon mais pas son hydroélectricité) ; elle trouve de larges utilisations en dehors de l'énergie électrique (en 1978, $10 \%$ seulement de la production du pétrole-gaz (1) et $43 \%$ de celle du charbon [1] ont été utilisés à la fourniture d'électricité) ; leur transformation en courant électrique néces-

(1) Chiffre calculé comme suit :

- gaz naturel et pétrole en $1978=6400$ Mtep (2) $\times 62 \%=$ 3970 Mtep [1].

- quantité d'électricité produite à partir du gaz et du pétrole : $7617 \mathrm{TWh}-[1660(\mathrm{H})+531(\mathrm{~N})+3766(\mathrm{C})]=1660 \mathrm{TWh}$ $[2]=370$ Mtep soit $9.3 \%$ de 3970 Mtep.

Le chiffre indiqué en 121 page 275 pour l'hydroélectricité a été porté de 1557 à 1660 TWh pour tenir compte d'une erreur possible sur le chiffre de production des USA. C'est par pure coincidence que l'on a le même chiffre pour l'hydraulique (H) que pour le pétrole + gaz $(P+G)$. site des investissements plus légers que pour les énergies renouvelables.

Le développement industriel des $\mathrm{XIX}^{\mathrm{e}}$ et $\mathrm{XX}^{\mathrm{e}}$ siècles s'est donc effectué d'abord par les énergies non-renouvelables : le charbon, grâce à la machine à vapeur, a ouvert la voie bien avant l'apparition de l'électricité.

Actuellement, et sans doute pour longtemps, les énergies non-renouvelables sont les grands de l'énergie : en 1978 , pétrole, gaz et charbon représentaient respectivement 43, 19 et $30 \%$ (total $92 \%$ ) de la production d'énergie [1].

L'énergie hydraulique, première actuellement des petits, doit son envol à l'électricité et c'est dans ce domaine que son importance est à apprécier plutôt que dans celui plus vaste de la production d'énergie où de larges secteurs lui sont fermés. C'est dans les seconds rôles qu'il faut chercher sa place, où elle remporte oscars et césars.

2. Mais cette appréciation n'est vraie qu'en moyenne dans certains pays, l'hydraulique joue un rôle de premier plan. Ainsi pour vingt d'entre eux, la part de l'hydraulique dans l'électricité $(\mathrm{H} / \mathrm{E})$ est supérieure à $50 \%$ (Tableaux III et IV).

Et il ne s'agit pas que de petits pays (Ghana, Zambie, Zaire, Mozambique, Zimbabwe, Egypte, Pérou, Colombie, Chili, Vénézuéla, Nouvelle-Zélande, Corée du Nord); il s'en trouve de très grands (Brésil, Canada) et beaucoup en Europe (Norvège, Portugal, Suisse, Autriche, Suède, Yougoslavie).

3. On ne peut pas traiter de la place de l'hydraulique du seul point de vue quantitatif.

Une usine nucléaire fait penser aux merveilles de la science, aux progrès de la technique alors qu'une usine 
hydroélectrique exprime la victoire de l'homme sur la nature : c'est l'exaltation de sa puissance, l'évocation d'un Prométhée délivré de ses liens. Ainsi, il y a une ou deux décennies seulement, les usines hydroélectriques des pays développés étaient moins des bâtiments industriels que des temples célébrant par leur architecture et leurs fresques victoire et délivrance, avec parfois des anges dans le ciel bleu du plafond (usine souterraine de Soverzerne, Piave, Italie). Pour les pionniers de l'époque de sa construction, Bratsk fut le symbole de la puissance russe, la vitrine de la Sibérie soviétique, une Jérusalem nouvelle chantée par le poête Evtouchenko. Au Québec "La Manic" est passée dans la légende, et à propos des travaux de la Baie James (Canada) et de l'Amazonie (Brésil) on parle de frontière reculée, de nouvelle frontière.

$\mathrm{Ce}$ culte se retrouve maintenant dans les pays en voie de développement (fresques aux usines de Moxoto et Paulo Afonso IV sur le rio San Francisco au Brésil).

Dans les zones habitées, dompter un fleuve assure le développement économique de toute une région : par delà la notion d'aménagements à buts multiples c'est le développement intégré d'une zone qui est visé. Faut-il rappeler l'exemple si souvent cité de la Tennessee
Valley Authority et d'autres moins connus comme le North of Scotland Hydro-Electric Board (NSHEB), créé en 1943, pour produire de l'électricité mais aussi pour assurer le développement économique et social de l'Ecosse (développement de la pêche, du tourisme, formation de tailleurs de pierre, construction de routes, de maisons. . .).

Est-ce trop dire que cette énergie hydraulique plus qu'une autre est l'objet d'un culte et relève du sacré ? Mais qu'on ne s'y trompe pas : c'est la victoire de l'homme sur lui-même qu'elle célèbre.

\section{Situation actuelle}

\section{Potentiel équipable}

Le potentiel équipable est estimé à 9800 TWh : c'est l'équivalent de la production mondiale d'énergie en 1955 ou de ce que sera la production mondiale d'électricité en 1983.

C'est aussi $27 \%$ du potentiel sauvage estimé à 36000 TWh (Tableau I) et même $33 \%$ si l'on considère le chiffre de 12000 TWh mentionné au chapitre : "Les facteurs d'incertitude".

\begin{tabular}{|c|c|c|c|c|c|c|}
\hline \multicolumn{7}{|c|}{ Tableau I. - Potentiel sauvage et potentiel équipable dans le monde par zones géographiques [4]. } \\
\hline \multirow{3}{*}{ Zones géographiques } & \multirow{3}{*}{$\begin{array}{l}\text { Superficie } \\
10^{6} \mathrm{~km}^{2}\end{array}$} & \multirow{3}{*}{$\begin{array}{c}\text { Potentiel (3) } \\
\text { sauvage } \\
\text { (TWh) }\end{array}$} & \multicolumn{4}{|c|}{ Potentiel équipable } \\
\hline & & & \multirow{2}{*}{$(G W)(4)$} & \multirow[b]{2}{*}{$(T W h)(4)$} & \multicolumn{2}{|c|}{ par $\mathrm{km}^{2}$} \\
\hline & & & & & $\left(\mathrm{MWh} / \mathrm{km}^{2}\right)$ & $\begin{array}{c}\text { rapport } \\
\text { à la moyenne }\end{array}$ \\
\hline Europe (sans I'URSS) & 4866 & 3400 & 215 & 700 & 140 & 2 \\
\hline URSS & 22272 & 4000 & 269 & 1100 & 50 & 0,7 \\
\hline $\begin{array}{l}\text { Etats-Unis } \\
\text { Canada, Groenland }\end{array}$ & 21498 & 6100 & 200 & 1300 & 60 & 0,85 \\
\hline Japon, Chine & 10370 & 9000 & 380 & 1450 & 140 & 2 \\
\hline $\begin{array}{l}\text { Amérique Centrale } \\
\text { Amérique du Sud }\end{array}$ & 20500 & 5400 & 328 & 1850 & 90 & 1,3 \\
\hline Afrique & 30300 & 6300 & 437 & 2000 & 66 & 0,94 \\
\hline $\begin{array}{l}\text { Asie sans le Japon } \\
\text { la Chine et la Sibérie }\end{array}$ & 20865 & $?$ & 309 & 1200 & 58 & 0,83 \\
\hline Océanie & 8521 & 1500 & 38 & 200 & 22,5 & 0,32 \\
\hline Antarctique & 14000 & 200 & - & - & - & - \\
\hline $\begin{array}{l}\text { Total arrondi } \\
\text { sans l'Antarctique }\end{array}$ & 140000 & 36000 & 2266 & 9800 & 70 & \\
\hline $\begin{array}{l}\text { (3) Certains de ces chiffre } \\
\text { 1974" (CME) [4] mais certair } \\
\text { non le potentiel sauvage qui es } \\
\text { (4) On peut retrouver ces } \\
\text { (Tableau VI.7) [4]. }\end{array}$ & $\begin{array}{l}\text { vent être retro } \\
\text { nnées de ce T } \\
\text { tioné. } \\
\text { es dans "Enque }\end{array}$ & $\begin{array}{l}\text { és au Tableau } V \\
\text { leau sont erroné } \\
\text { sur les ressource }\end{array}$ & $\begin{array}{l}\text { page } 171 \mathrm{~d} \\
\text { nergétiques }\end{array}$ & $\begin{array}{l}\text { olume "Enqu } \\
\text { sour les Etat } \\
\text { 4" (CME) [4 }\end{array}$ & $\begin{array}{l}\text { sur les ressourc } \\
\text { nis c'est le pote } \\
\text { Dages } 187 \text { et suiv }\end{array}$ & $\begin{array}{l}\text { es énergétiques - } \\
\text { ntiel équipable et } \\
\text { antes et page } 173\end{array}$ \\
\hline
\end{tabular}




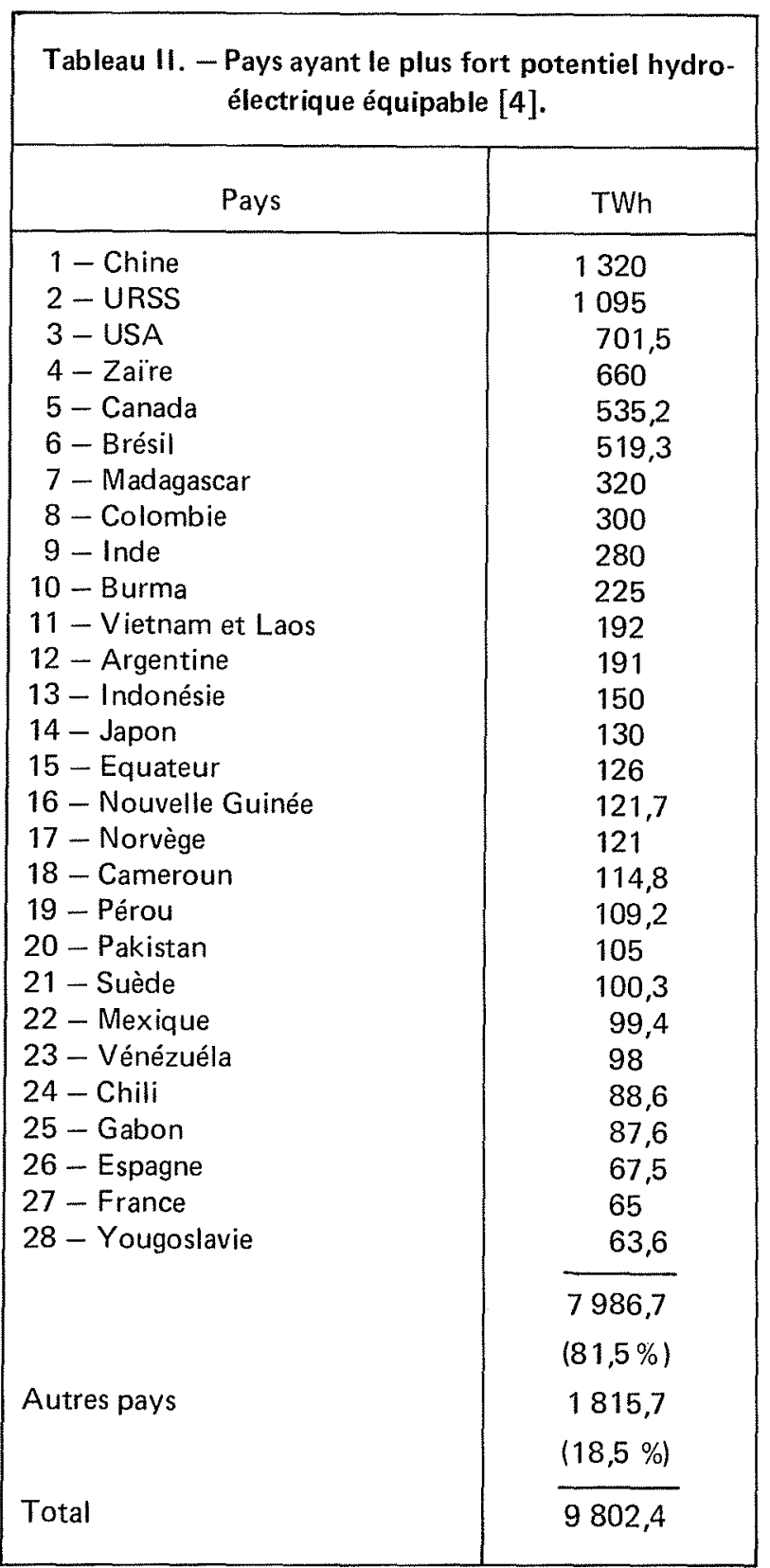

Ces chiffres sont extraits de la dernière publication de la Conférence Mondiale de l'Energie (CME) sur ce sujet [4]. Des documents ultérieurs de la CME ont repris ces chiffres [5], parfois avec des erreurs [6]. Certaines publications citent des chiffres largement divergents parfois dans le même ouvrage [7].

La diversité des présentations et des unités employées peuvent expliquer ces chiffres contradictoires. Le potentiel équipable est en effet exprimé :

- tantôt en énergie annuelle (en TWh ou en TJ ou en Gtep) :

$$
\begin{array}{rrr}
9800 & \text { TWh; }\left(1 \mathrm{TWh}=10^{12} \mathrm{Wh}=10^{9} \mathrm{kWh}\right) \\
-35921000 & \text { TJ } & (1 \mathrm{TWh}=3600 \mathrm{TJ}) \\
- & 2.2 \text { Gtep } & (1 \mathrm{Gtep}=4500 \mathrm{TWh})
\end{array}
$$

- tantôt en puissance

- 2200 GW ou 2.2 TW avec. une durée d'utilisation de cette puissance de $4380 \mathrm{~h}(8760 / 2)$ soit avec un coefficient d'utilisation de 0,5 ;
- en puissance annuelle moyenne équivalente (en MW) c'est-à-dire la productibilité divisée par $8760 \mathrm{~h}$, celle-ci pouvant être la productibilité annuelle moyenne ou la productibilité garantie (firm energy) (5).

\section{Les facteurs d'incertitude}

Le potentiel équipable n'est pas une donnée certaine. Son estimation est variable car entachée d'incertitudes qui ont théoriquement trait, pour chaque pays ;

- au contexte économique (coût des autres énergies, taux d'actualisation) ;

- à l'environnement social (acceptation ou refus des submersions, des détournements de rivières),

- à la volonté politique de mise en œuvre de l'hydroélectricité

- et à la possibilité d'aménagements à buts multiples.

En pratique la fiabilité du potentiel dépend des moyens mis en œuvre pour l'estimer.

Ainsi en France, pendant ces dernières trente années, les estimations ont varié entre 60 et 80 TWh ; l'estimation actuelle est de 72 TWh, dont 66 TWh (92\%) en service et en construction au $1^{\text {er }}$ janvier 1982 .

Pour les plus grands pays en voie de développement, l'incertitude est plus grande encore : par exemple, les estimations viennent de passer de 1300 à 1900 TWh pour la Chine et de 500 à $800 \mathrm{TWh}$ pour le Brésil.

En général, elles sont corrigées en hausse quand le seul point de vue économique est en jeu ; les contraintes écologiques pourraient provoquer une légère réduction. A $9800 \mathrm{TWh}$, le potentiel hydroélectrique serait plutôt sous-estimé : 12000 TWh serait un chiffre plus probable. Dans ce qui suit, on conservera néanmoins pour le Brésil, la Chine, la France, les chiffres cités dans l'Enquête de la CME qui a été publiée en 1974 [4].

\section{Sa distribution dans le monde}

La répartition par zones- géographiques est donnée au Tableau I.

Elle n'est pas proportionnelle à la surface, car le relief et la pluviométrie introduisent des différences. A l'intérieur de chacune des grandes zones, la distribution n'est pas non plus uniforme.

A ce titre, l'Afrique est un cas extrême : son potentiel (2000 TWh) est concentré sur la bordure orientale montagneuse et la zone équatoriale ; de plus, la moitié intéresse deux pays seulement, Zaire $(660 \mathrm{TWh})$ et Madagascar (320 TWh) (6) et de surcrôt, au Zaïre, la moitié (300 TWh) se retrouve en un seul site, Inga.

Malgré ces inégalités l'énergie hydraulique est mieux répartie dans le monde que le charbon, le pétrole, le gaz et même l'uranium.

Le Tableau II donne la liste des pays ayant le plus fort potentiel équitable.

(5) Voir à ce sujet le Tableau introductif sur le potentiel du Brésil donné plus loin dans ce numéro de la Houille Blanche par C.A. Amarante dans son article sur le Parana.

(6) Madagascar $-587041 \mathrm{~km}^{2}$ : le potentiel équipable est, au $\mathrm{km}^{2}$, 4 fois celui de la France, la moitié de celui de la Suisse. 


\section{Potentiel équipé en 1978 (7)}

\section{Dans le monde}

En 1978, la part équipée du potentiel était de l'ordre de 1660 TWh soit 370 Mtep, en assimilant la productibilité à la production de cette année-là.

Elle représentait $17 \%$ du potentiel équipable (9800 TWh) et $21,7 \%$ de la production d'électricité (7617 TWh).

La figure 1 montre les parts respectives des quatre grandes sources primaires d'électricité : la contribution de 1'hydraulique est égale pour 1978, à celles réunies du pétrole et du gaz. Cette appréciation est plus significative du rôle de l'hydraulique que de celle donnant seulement la part de l'hydraulique dans la production d'énergie $(5,5 \%)(8)$.

On peut également noter que l'hydraulique a représenté $19 \%$ de la production charbonnière et que les 7617 TWh d'électricité auraient pu être obtenus par $87 \%$ de la production charbonnière de cette année là.

Les Tableaux III et IV donnent la liste des principaux pays producteurs d'hydroélectricité. Deux points sont à souligner :

- pour vingt pays, la part de l'hydraulique dans l'électricité est supérieure à $50 \%$;

- 15 seulement des 28 premiers pays pour le potentiel équipable (Tableau II) figurent parmi les 24 pays qui fournissent $90 \%$ du potentiel équipé : La mise en valeur des ressources hydrauliques d'un pays n'est pas fonction de leur importance mais du degré de développement du pays.

La grande artère hydroélectrique est actuellement la Columbia (77.6 TWh en 1981 pour $18535 \mathrm{MW}$ installés) suivie par le St Laurent (52 TWh en 3 usines). La Grande (phase 1) prendra bientôt la $3^{\mathrm{e}}$ place avec $62 \mathrm{TWh}$, mais le Parana, après la mise en service d'Itaipu (70 TWh pour $12700 \mathrm{MW}$ de puissance installée) viendra en tête en 1988 avec 108 TWh (20 TWh sont actuellement en service à Ilha Solteira et Jupia et 88 en construction dont 70 à Itaipu). Encore ne s'agit-il que du Parana à l'aval du confluent Paranaiba-Grande où il prend le nom de Parana (50 TWh sont en service à l'amont).

\section{En France}

Au $1^{\text {er }}$ janvier 1982 , l'équipement du potentiel se présentait comme suit :

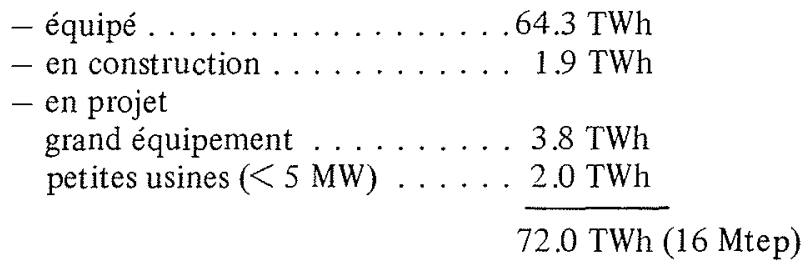

(7) Chiffres de production (et non de productibilité) pour l'année 1978 - tirés de [2].

(8) En 1978, la production d'électricité a représenté $25,6 \%$ de la production d'énergie ; la part de l'hydraulique dans l'énergie a donc été de $21,7 \% \times 25,6 \%=5,5 \%$.

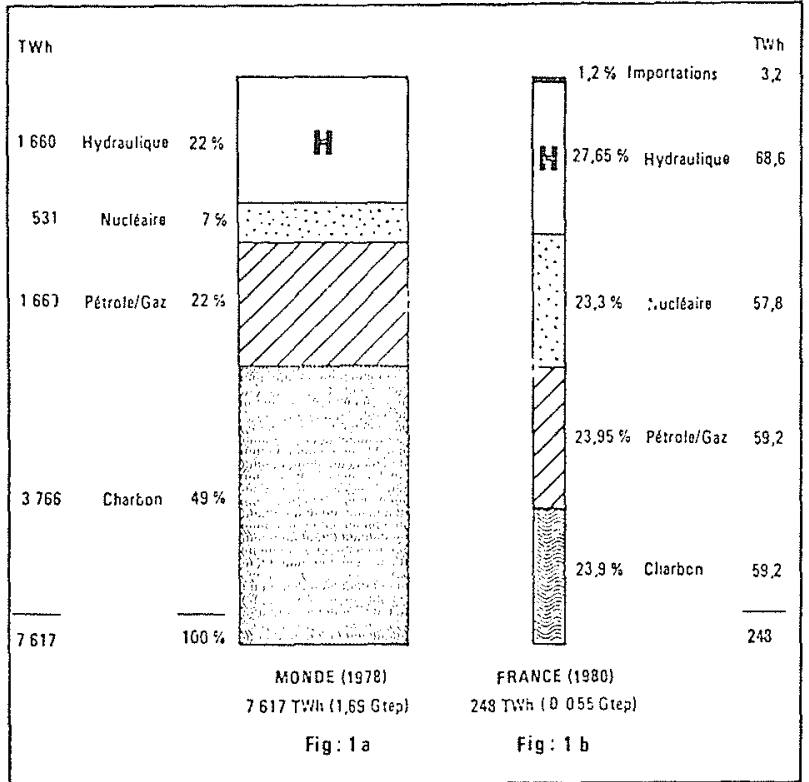

Figure 1 - Consommation d'électricité en 1978 dans le monde, en 1980 en France.

En 1981, la part équipée $(64.3 \mathrm{TWh})$ a représenté $89.3 \%$ du potentiel équipable et $27,4 \%$ de la production d'électricité (264 TWh).

En 1980, la figure 1 montre la situation tout à fait remarquable des parts respectives des quatre grandes sources primaires d'électricité : leur contribution est sensiblement d'un quart pour chacune d'elles.

En 1981, la poussée du nucléaire se fait au détriment du pétrole-gaz, mais laisse intacte la part de l'hydraulique dans la consommation (Tableau V).

Pendant les dix dernières années, le rapport $\mathrm{H} / \mathrm{E}$ a évolué comme indique dans le Tableau VI dans lequel : - la $1^{\text {ère }}$ ligne correspondant pour $\mathrm{H}$ à la production, - la $2^{\mathrm{e}}$ ligne à la productibilité en année d'hydraulicité moyenne ;

- le coefficient d'hydraulicité est donné en $3^{\mathrm{e}}$ ligne : la bonne hydraulicité depuis cinq ans masque la réduction de la place de l'hydraulique dans le parc de nos moyens de production.

En France, en 1980, compte tenu de la régression du charbon, l'hydroélectricité (15.5 Mtep) est la première des énergies primaires nationales devant le charbon (15.1 Mtep) [8], le pétrole et le gaz (9.2 Mtep) [8] puis le nucléaire (6.4 Mtep) (9). Il en a été de même en 1981, mais en 1982, charbon et nucléaire devanceront l'hydroélectricité.

\section{Répartition mondiale}

Du point de vue du développement économique, on peut distinguer actuellement trois groupes de pays :

(9) En comptant le nucléaire pour moitié (minerai métropolitain). L'ensemble du nucléaire est souvent compté comme une source primaire nationale, l'autre moitié du minerai provenant de zones considérées stratégiquement sûres. 
- les pays développés, c'est-à-dire les 24 pays de l'OCDE : leur hydroélectricité a été mise en cuvre au même titre que leurs autres ressources énergétiques - cinq pays (URSS, Inde, Brésil, Mexique, Yougoslavie) très largement en tête des pays en voie de développement : leur équipement hydroélectrique est avancé, mais moins que dans les pays précédents
- les autres pays dont le développement ne fait que commencer : leur potentiel équipable $(55 \%$ du potentiel mondial) y est quasi intact ( $4 \%$ est équipé)

Les caractéristiques de ces trois groupes sont données au Tableau VII qui montre à l'évidence le lien étroit qui existe entre développement économique, production d'électricité et équipement du potentiel hydroélec-

Tableau III. - Part de I'hydraulique dans la production d'électricité en 1978, pour les principaux pays producteurs d'hydroélectricité. Par ordre décroissant de production d'hydroélectricité [2].

\begin{tabular}{|c|c|c|c|}
\hline Pays & $\begin{array}{c}\text { Hydroélectricité } \\
\text { H(TWh) }\end{array}$ & $\begin{array}{c}\text { Electricité totale } \\
E(T W h)\end{array}$ & $H / E \%$ \\
\hline $\begin{array}{l}1 \text { - USA } \\
2 \text { - Canada } \\
3 \text { - URSS } \\
4 \text { - Brésil } \\
5 \text { - Norvège } \\
6 \text { - Japon } \\
7 \text { - France } \\
8 \text { - Chine } \\
9 \text { - Italie } \\
10 \text { - Suède } \\
11 \text { - Espagne } \\
12 \text { - Inde } \\
13 \text { - Suisse } \\
14 \text { - Autriche } \\
15 \text { - Yougoslavie } \\
16 \text { - Mexique } \\
17 \text { - Corée du Nord } \\
18 \text { - Allemagne Fédérale } \\
19 \text { - Nouvelle-Zélande } \\
20 \text { - Australie } \\
21 \text { - Finlande } \\
22 \text { - Vénézuéla } \\
23 \text { - Colombie } \\
24 \text { - Portugal } \\
25 \text { - Egypte } \\
26 \text { - Turquie } \\
27 \text { - Roumanie } \\
28 \text { - Zambie } \\
29 \text { - Mozambique } \\
30 \text { - Chili } \\
31 \text { - Pérou } \\
32 \text { - Argentine } \\
33 \text { - Royaume-Uni } \\
34 \text { - Philippines } \\
35 \text { - Pakistan } \\
36 \text { - Tchécoslovaquie } \\
37 \text { - Ghana } \\
38 \text { - Iran } \\
39 \text { - Zaire } \\
40 \text { - Zimbabwe } \\
41 \text { - Bulgarie } \\
\text { - }\end{array}$ & $\begin{array}{l}306 \\
230 \\
159 \\
103 \\
78 \\
76 \\
73 \\
67 \\
53 \\
53 \\
44 \\
39 \\
32 \\
25 \\
25 \\
21 \\
19,5 \\
19 \\
17 \\
15 \\
13 \\
12,75 \\
10,5 \\
10 \\
9,2 \\
9,2 \\
8,9 \\
8,5 \\
6,75 \\
6,7 \\
6,35 \\
6 \\
5,5 \\
5,2 \\
4,9 \\
4,4 \\
4,25 \\
4 \\
4 \\
3,5 \\
3,5 \\
1601,60 \\
658 \mathrm{TWh}\end{array}$ & $\begin{array}{c}2328 \\
327 \\
1190,5 \\
110,6 \\
78,6 \\
565 \\
218,2 \\
225 \\
173,4 \\
89 \\
100,3 \\
107 \\
43,5 \\
38,2 \\
50,5 \\
53,6 \\
32 \\
352,1 \\
21,6 \\
86 \\
33,4 \\
24,3 \\
15,5 \\
13,1 \\
13,5 \\
21,2 \\
61,6 \\
8,7 \\
7,2 \\
10,2 \\
8,9 \\
34 \\
288,9 \\
17 \\
11 \\
68,9 \\
4,3 \\
18,5 \\
4,1 \\
3,9 \\
29,7 \\
6887,90 \\
90 \% \\
617 \text { TWh }\end{array}$ & $\begin{array}{r}13,1 \\
70,3 \\
13,3 \\
93,1 \\
99,2 \\
13,4 \\
33,4 \\
29,8 \\
30,6 \\
59,5 \\
43,9 \\
36,4 \\
73,6 \\
65,4 \\
49,5 \\
39,2 \\
60,9 \\
5,4 \\
78,7 \\
17,4 \\
38,9 \\
52,5 \\
67,7 \\
76,3 \\
68,1 \\
43,4 \\
14,4 \\
97,7 \\
93,8 \\
65,7 \\
71,3 \\
17,6 \\
1,9 \\
30,6 \\
44,5 \\
6,4 \\
98,8 \\
21,6 \\
97,6 \\
89,7 \\
11,8 \\
23,2\end{array}$ \\
\hline
\end{tabular}


Tableau IV. - Part de I'hydraulique dans la production d'électricité en 1978, pour les principaux pays producteurs d'hydroélectricité. Par ordre décroissant de H/E [2]

\begin{tabular}{|c|c|}
\hline Pays & H/E \% \\
\hline Norvège & 99,2 \\
\hline Ghana & 98,8 \\
\hline Zambie & 97,7 \\
\hline Zaire & 97,6 \\
\hline Mozambique & 93,8 \\
\hline Brésil & 93,1 \\
\hline Zimbabwe & 89,7 \\
\hline Nouvelle-Zélande & 78,7 \\
\hline Portugal & 76,3 \\
\hline Suisse & 73,6 \\
\hline Pérou & 71,3 \\
\hline Canada & 70,3 \\
\hline Egypte & 68,1 \\
\hline Colombie & 67,7 \\
\hline Chili & 65,7 \\
\hline Autriche & 65,4 \\
\hline Corée du Nord & 60,9 \\
\hline Suède & 59,5 \\
\hline Vénézuéla & 52,5 \\
\hline Yougoslavie & 49,5 \\
\hline Pakistan & 44,5 \\
\hline Espagne & 43,9 \\
\hline Turquie & 43,4 \\
\hline Mexique & 39,2 \\
\hline Finlande & 38,9 \\
\hline Inde & 36,4 \\
\hline France & 33,4 \\
\hline Italie & 30,6 \\
\hline Philippines & 30,6 \\
\hline Chine & 29,8 \\
\hline Iran & 21,6 \\
\hline Argentine & 17,6 \\
\hline Australie & 17,4 \\
\hline Roumanie & 14,4 \\
\hline Japon & 13,4 \\
\hline URSS & 13,3 \\
\hline USA & 13,1 \\
\hline Bulgarie & 11,8 \\
\hline Tchécoslovaquie & 6,4 \\
\hline Allemagne Fédérale & 5,4 \\
\hline Royaume-Uni & 1,9 \\
\hline
\end{tabular}

\begin{tabular}{|l|c|c|}
\hline \multicolumn{3}{|c|}{$\begin{array}{c}\text { Tableau V - Parts respectives des quatre grandes } \\
\text { sources primaires d'électricité è France }\end{array}$} \\
\hline \multicolumn{1}{|c|}{$\begin{array}{l}\text { Electricité } \\
\text { d'origine }\end{array}$} & 1980 & 1981 \\
Hydraulique & $27,65 \%$ & $28 \%$ \\
Nucléaire & $23,3 \%$ & $38,5 \%$ \\
Pétrole + Gaz & $23,95 \%$ & $13,4 \%$ \\
Charbon & $23,9 \%$ & $22,2 \%$ \\
& $98,8 \%$ & $102,1 \%$ \\
Importations & $+1,2 \mathrm{TWh}$ & \\
Exportations & & $-2,1 \%$ \\
Production & $245 \mathrm{TWh}$ & $264 \mathrm{TWh}$ \\
Consommation & $248 \%$ & $258 \%$ \\
\hline
\end{tabular}

trique : sur les $17 \%$ équipés du potentiel, $11,5 \%$ se trouvent dans les pays de l'OCDE soit les $2 / 3$.

Le détail pour les pays de l'OCDE est donné au Tableau VIII.

\section{Perspectives d'avenir}

Modifications attendues dans la répartition du potentiel équipé

Le développement de l'hydroélectricité n'est pas conditionné par la concurrence du charbon ou du nucléaire. Là où existe un potentiel équipable, économiquement et écologiquement parlant, sa mise en valeur va de pair avec le développement économique du pays : les besoins en capitaux sont le frein commun.

Il faut donc s'attendre à trouver dans 20 ans, en l'an 2000 , quatre groupes de pays représentatifs de quatre stades d'équipement du potentiel hydraulique, comme indiqué au Tableau IX.

On arriverait ainsi, en l'an 2000, à un maximum équipé de 3500 TWh représentant $35 \%$ du potentiel équipable, soit un doublement en 22 ans, ce qui correspond à un taux d'accroissement moyen de $3,5 \%$. Cet équipement représenterait aussi $15 \%$ de la production d'électricité et $6,5 \%$ de la production d'énergie.

$\mathrm{La}$ résistance des écologistes pourrait réduire le montant du potenticl ćquipablc par lc gel (momentané ?) de certains sites -en Suède par exemple, $16 \mathrm{TWh}$ sur quatre grandes rivières sont exclus, de par la loi, de toute

\begin{tabular}{|c|c|c|c|c|c|c|c|c|c|}
\hline \multicolumn{10}{|c|}{ Tableau VI } \\
\hline Années & 1973 & 1974 & 1975 & 1976 & 1977 & 1978 & 1979 & 1980 & 1981 \\
\hline $\begin{array}{l}\mathrm{H} / \mathrm{E} \% \\
\text { en production }\end{array}$ & 27,2 & 31,7 & 33,5 & 24 & 37,6 & 31,5 & 29 & 28,3 & 27,4 \\
\hline $\begin{array}{l}\mathrm{H} / \mathrm{E} \% \\
\text { en productibilité }\end{array}$ & 33 & 32,5 & 33 & 31 & 30 & 29 & 27 & 26 & 24 \\
\hline $\begin{array}{l}\text { Coefficient } \\
\text { d'hydraulicité }\end{array}$ & 0,85 & 0,99 & 1,01 & 0,83 & 1,28 & 1,02 & 1,13 & 1,12 & 1,15 \\
\hline
\end{tabular}


Tableau VII. - Répartition mondiale du potentiel équipé en 1978 [2].

\begin{tabular}{|c|c|c|c|c|c|c|c|}
\hline & \multirow{2}{*}{$\begin{array}{c}\text { Population } \\
\text { en } 1977 \\
\text { M hab. }\end{array}$} & \multirow{2}{*}{$\begin{array}{c}\text { Potentiel } \\
\text { équipable } \\
\text { TWh }\end{array}$} & \multicolumn{3}{|c|}{ Potentiel équipé } & \multirow{2}{*}{$\begin{array}{c}\text { Electricité } \\
\text { TWh }\end{array}$} & \multirow{2}{*}{$\begin{array}{c}\text { Hydro- } \\
\text { électricité } \\
\%\end{array}$} \\
\hline & & & TWh & $\begin{array}{l}\% \text { du potentiel } \\
\text { equipable }\end{array}$ & $\begin{array}{l}\% \text { du potentiel } \\
\text { mondial }\end{array}$ & & \\
\hline \multirow{4}{*}{$\begin{array}{l}\text { 1) OCDE } \\
\text { 2) URSS - } \\
\text { Inde Mexique- } \\
\text { Brésil } \\
\text { Yougoslavie } \\
\text { 3) Autres } \\
\text { pays (dont la } \\
\text { Chine) }\end{array}$} & 750 & 2100 & 1063 & $50 \%$ & $10,95 \%$ & 4946 & $21,4 \%$ \\
\hline & 1100 & 2100 & 348 & $16,5 \%$ & $3,55 \%$ & 1530 & $22,6 \%$ \\
\hline & 2250 & 5600 & 253 & $3,7 \%$ & $2,50 \%$ & 1231 & $16 \%$ \\
\hline & 4100 & 9800 & 1664 & & 17 & 7617 & $21.7 \%$ \\
\hline
\end{tabular}

Tableau VIII. - L'hydroélectricité dans les pays de l'OCDE. Potentiel et production en 1978 en TWh [2].

\begin{tabular}{|l|c|c|}
\hline \multicolumn{1}{|c|}{ Pays } & $\begin{array}{c}\text { Potentiel } \\
\text { équipable }\end{array}$ & $\begin{array}{c}\text { Production } \\
\text { en } 1978\end{array}$ \\
\hline 1 - USA & 701,5 & 304 \\
2 - Canada & 535,2 & 229,8 \\
3 - Australie & 24,5 & 14,5 \\
4 - Nouvelle-Zélande & 55,2 & 16,6 \\
5 - Japon & 130 & 75,8 \\
6 - Allemagne & 21,8 & 19 \\
7 - Autriche & 43,9 & 25,1 \\
8 - Belgique & 0,5 & 0,5 \\
9 - Danemark & - & 0,02 \\
$10-$ Espagne & 67,5 & 43,6 \\
11 - Finlande & 18 & 13,4 \\
12 - France & 65 & 73,4 \\
13 - Grèce & 15,6 & 1,93 \\
14 - Irlande & 0,9 & 1,05 \\
15 - Islande & 35 & 2,63 \\
16 - Italie & 50,6 & 52,7 \\
17 - Luxembourg & - & 0,3 \\
18 - Norvège & 121 & 78,35 \\
19 - Pays-Bas & - & - \\
20 - Portugal & 17,8 & 9,56 \\
21 - Royaume-Uni & 4,2 & 5,5 \\
22 - Suède & 100,3 & 52,7 \\
23 - Suisse & 32 & 34 \\
24 - Turquie & 65,3 & 9,2 \\
& 2105,8 & 1063,6 \\
& & \\
\hline
\end{tabular}

utilisation- ; mais elle ne devrait pas modifier sensiblement ce scénario, basé sur un taux de développement de $3 \%$ pour l'économie et de $2,5 \%$ pour l'énergie.

Illustrons cet effort par l'exemple d'un petit pays européen, le Portugal. A fin 1981 il reste à équiper $40 \%$ du potentiel en énergie et $60 \%$ en puissance ; 20 à 23 barrages pourraient être construits d'ici 2010 .

\section{Transport}

Les longues distances constitueront-elles un frein au développement de l'hydroélectricité, c'est-à-dire à l'équipement de chutes fort éloignées des centres de consommation?
Il semble qu'il n'en sera rien : les grandes distances auxquelles on est confronté actuellement sont de l'ordre de 800 à $1500 \mathrm{~km}$ et l'on ne devrait pas avoir à envisager, dans l'avenir, des distances supérieures à $3000 \mathrm{~km}$; de plus une solution économique en courant continu à haute tension plutôt qu'en courant alternatif triphasé est en train de s'imposer grâce aux progrès de l'électronique par la mise au point de thyristors de grande puissance [9] [10]. Un projet est à l'étude avec cette technique pour transporter sur $2400 \mathrm{~km}$, de Sibérie Centrale à Moscou, $6000 \mathrm{MW}$ sous $\pm 750 \mathrm{kV}$. Citons parmi ceux en construction : la liaison ItaipuSao Paulo au Brésil ( $800 \mathrm{~km}-6300 \mathrm{MW})$ et parmi ceux en service, celle de Cabora Bassa-Apollo (1414 km $1440 \mathrm{MW}$ ) entre le Mozambique et l'Afrique du Sud.

Pour fixer les idées, le coût du transport est de l'ordre de 2 centimes pour les convertisseurs d'extrêmité, auxquels il faut ajouter 2 centimes par tranche de $1000 \mathrm{~km}$ soit environ 8 centimes pour $3000 \mathrm{~km}$, pour une énergie de base. Au Canada, pour $1400 \mathrm{~km}$ de ligne, le transport en courant alternatif de l'énergie des usines de la Baie James jusqu'à Montréal majore le prix de revient de $\mathrm{kWh}$ de $50 \%$.

\section{Microcentrales [11]}

Pour résoudre la crise énergétique, elles font parfois figure de panacée auprès des non-initiés. Or leur appoint est et restera très marginal par rapport à la contribution de l'hydraulique, elle-même relativement limitée.

En France, ces installations sont particulièrement nombreuses : à fin 1979, il y avait en service 1170 usines de puissance installée inférieure à $5000 \mathrm{~kW}$ (11), dont 217 exploitées par EDF, produisant 3,5 TWh contre $2,8 \mathrm{TWh}$ en 1964 ; il n'est pas exclu d'arriver à un total de 5,5 à 6 TWh vers $1990-2000$ ce qui représenterait $8 \%$ de la production hydroélectrique française (fig. 2).

(11) 1060 usines de puissance installée inférieure à $2000 \mathrm{~kW}$, produisant $1,6 \mathrm{TWh}$ soit $2,5 \%$ de la production actuelle. C'est généralement à ces usines $(\mathrm{Pi}<2000 \mathrm{KW}$ ) qu'est réservé le nom de microcentrales. 


\begin{tabular}{|c|c|c|c|c|c|}
\hline \multicolumn{6}{|c|}{ Tableau IX. - Répartition mondiale estimée du potentiel équipé en 2000.} \\
\hline & $\begin{array}{c}\text { Taux } \\
\text { d'accroissement } \\
\text { du potentiel }\end{array}$ & $\begin{array}{l}\text { Potentiel } \\
\text { équipable }\end{array}$ & \multicolumn{3}{|c|}{ Potentiel équipé en 2000} \\
\hline & $\%$ & TWh & $\begin{array}{l}\% \text { du potentiel } \\
\text { équipable }\end{array}$ & TWh & $\begin{array}{c}\% \text { du potentiel } \\
\text { mondial }\end{array}$ \\
\hline 1) $O C D E$ & $1,8 \%(10)$ & 2100 & $50 \% \times 1,45=72 \%$ & 1500 & $15 \%$ \\
\hline $\begin{array}{l}\text { 2) URSS - Inde } \\
\text { Mexique - Brésil } \\
\text { Yougoslavie }\end{array}$ & $5,6 \%$ & 2100 & $16,5 \% \times 3,33=55 \%$ & 1150 & $11,5 \%$ \\
\hline $\begin{array}{l}\text { 3) Un groupe en début } \\
\text { de développement }\end{array}$ & $7 \%$ & 2600 & $4,7 \% \times 4,4=21 \%$ & 550 & $5,5 \%$ \\
\hline \multirow[t]{2}{*}{$\begin{array}{l}\text { 4) Un groupe en attente } \\
\text { de développement }\end{array}$} & $3,5 \%$ & 3000 & $4,7 \% \times 2,1=10 \%$ & 300 & $3,0 \%$ \\
\hline & $3,5 \%$ & 9800 & & 3500 & $35 \%$ \\
\hline
\end{tabular}

(10) $2,2 \%$ pour USA-Canada.

Les zones à l'écart des grandes voies de desserte, dans les pays en voie de développement, ont vocation à être équipées de microcentrales hydrauliques plutôt que de diésels quand l'eau et la chute y sont disponibles. Mais les grandes sociétés distributrices d'électricité ne sont pas motivées par cet équipement de détail et un organisme national chargé de l'équipement et de la maintenance fait souvent défaut ! Et c'est paradoxalement dans les pays développés que se trouve le marché le plus actif de petits groupes pour microcentrales : le confort (chauffage économique des locaux) ou le profit (en France, rachat obligatoire par EDF) sont les motivations essentielles.

\section{Centrales de pompage}

Les centrales de transfert d'énergie par pompage utilisent l'eau en circuit fermé entre deux bassins, en vue de la fourniture d'électricité aux heures de pointe. Il ne s'agit plus de la mise en œuvre d'une énergie primaire mais du report d'une énergie d'heures creuses (en général thermique) pour disposer d'une puissance de pointe à moindre coût qu'avec une nouvelle centrale thermique.

Leur justification est triple :

- souplesse d'adaptation à la charge : celle des usines hydrauliques à réservoir ;

- grande puissance installée : on arrive à $1000 \mathrm{MW}$ de puissance installée avec des bassins de $7 \mathrm{hm}^{3}$ (12) et une dénivelée de $250 \mathrm{~m}$;

- coût total actualisé, fonction du site (hauteur de chute et éloignement des deux bassins) inférieur à

(12) $1 \mathrm{hm}^{3}=(100 \mathrm{~m})^{3}=10^{6} \mathrm{~m}^{3}$.

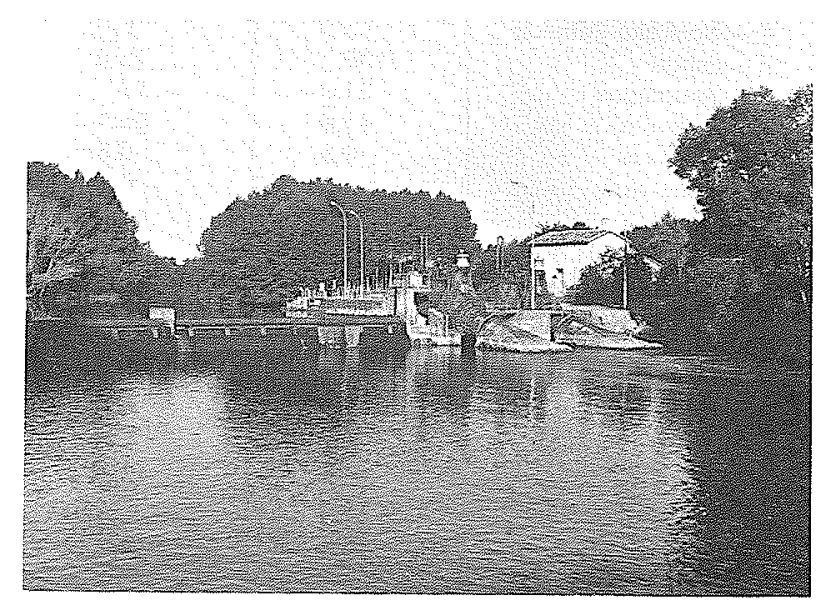

Figure 2 - Microcentrale sur la Dordogne. Groupe en siphon.

celui d'une usine thermique équivalente, fonctionnant à un rendement affaibli par celui du cycle pompageturbinage $(0,72)$.

Leur développement, qui date de 1960 est remarquable : leur puissance installée s'élève déjà à $43 \mathrm{GW}$ en 1978 , soit $10,5 \%$ de la puissance installée totale (408 GW en 1978) des usines hydrauliques classiques les centrales en construction représentent $28 \mathrm{GW}$.

En France il y a $2 \mathrm{GW}$ de pompage en service en 1980 pour $20 \mathrm{GW}$ en hydraulique classique $(10 \%)$; 2,8 GW sont en construction (fig. 3 ).

\section{Taille des aménagements hydroélectriques}

Deux raisons conduisent à des aménagements hydroélectriques à très forte puissance installée. 
a) L'équipement des grands fleuves de plaine met en jeu des débits considérables.

On compte déjà dans le monde (Amérique et URSS) 20 usines de plus de $2000 \mathrm{MW}$. La plus puissante est celle de Grand Coulée sur la rivière Columbia avec $10230 \mathrm{MW}$. A Itaipu (Brésil) on installe actuellement $12700 \mathrm{MW}$ avec possibilité d'un suréquipement à $21500 \mathrm{MW}$; la production sera de $70 \mathrm{TWh}$, soit tout le potentiel équipable français concentré en une chute de $120 \mathrm{~m}$.

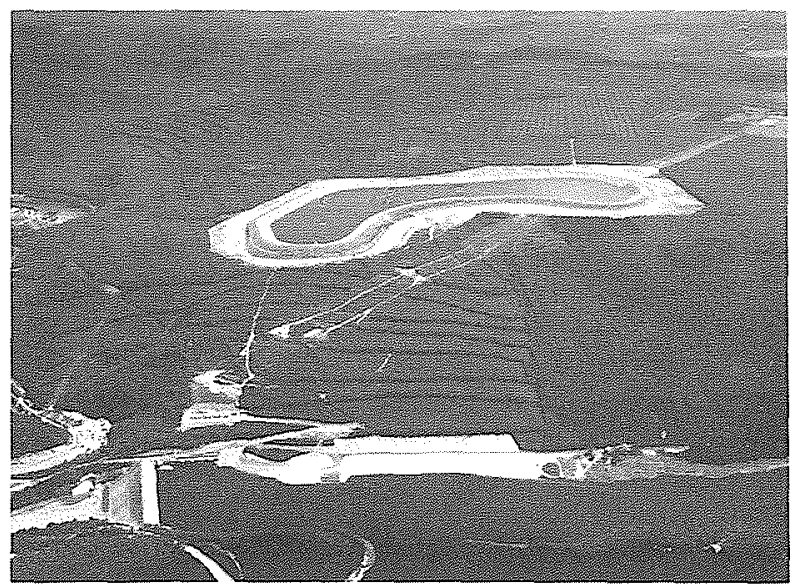

Figure 3 - La centrale de pompage de Revin. Vue d'ensemble des deux bassins.

En Europe, l'usine d'Aldéadavilla (Portugal) mise en service en 1963 sur le cours international du Douro, a été l'usine la plus puissante d'Europe (762 MW-
2440 GWh) jusqu'à la mise en service de l'usine des Portes de Fer (sur le Danube à la frontière de la Roumanie et de la Yougoslavie) (2050 MW-11.4 TWh).

b) Dans les pays ayant atteint un niveau minimal de développement et où l'hydroélectricité ne représente pas plus de 20 à $30 \%$ de la production d'électricité, les centrales hydrauliques de réservoir sont de plus en plus suréquipées en vue de la fourniture d'énergie de pointe ( 6 h par jour), l'électricité de base étant produite par d'autres moyens.

Dans les pays en voie de développement, l'aménagement de Guri sur le Caroni au Vénézuéla est un bel exemple de suréquipement avec comme étapes : $600 \mathrm{MW}$ en $1969-2000 \mathrm{MW}$ en $1978-10000 \mathrm{MW}$ en 1986 , la dernière étape comportant une surélévation de 100 à $160 \mathrm{~m} \mathrm{du}$ barrage ; capacité du réservoir : $135 \mathrm{~km}^{3}$ pour des apports annuels moyens de $159 \mathrm{~km}^{3}$ soit $5000 \mathrm{~m}^{3} / \mathrm{s}$ ou $60 \mathrm{l} / \mathrm{s} / \mathrm{km}^{2}$ (bassin versant de 85000 $\mathrm{km}^{2}$ ).

La phase finale de l'équipement de Grand Coulée (usine de tête de la Columbia) sera aussi de $10000 \mathrm{MW}$. Itaipu, dans sa phase actuelle $(12700 \mathrm{MW})$ est une usine au fil de l'eau.

Evolution comparée des productions d'énergie, d'électricité et d'hydroélectricité (Tableau X).

La production d'énergie primaire s'est développée de 1950 à 1974 au taux quasi constant de $5 \%$ contre $2,2 \%$ pour la période de 1925-1950; celui-ci est tombé à 1,17 de 1974 à 1978 ; on escompte une valeur future de $2,5 \%$ pour un taux de croissance économique de $3 \%$.

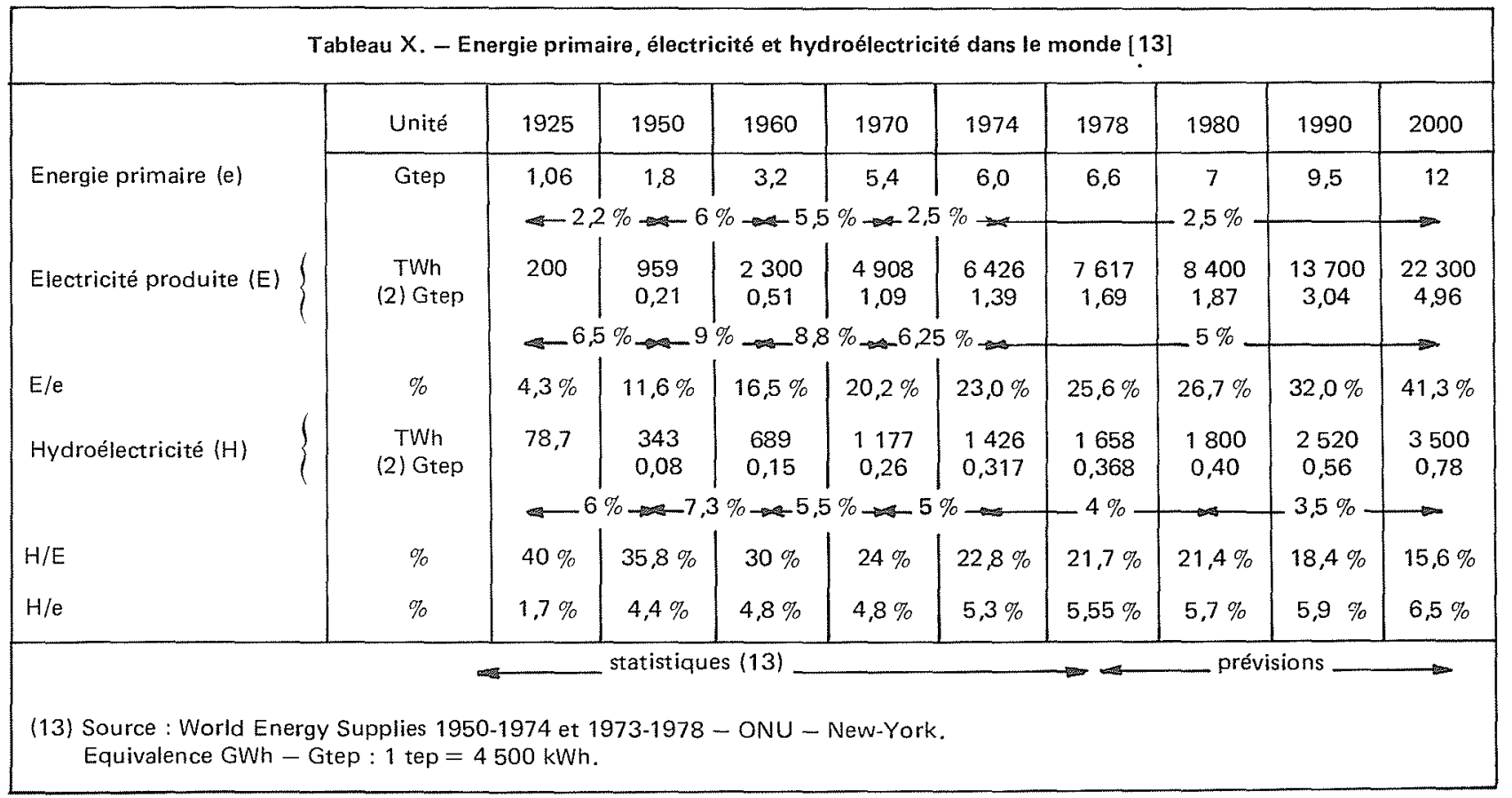


La production d'électricité de 1950 à 1963 s'est accrue au taux de $9 \%$ contre $6,5 \%$ de 1925 à 1950 ; celui-ci s'est progressivement réduit pour n'être plus que de $5 \%$ de 1974 à 1978 ; on admet qu'il se maintiendra à cette valeur dans les 20 ans qui viennent.

La croissance plus rapide de l'électricté, comparée à celle de l'énergie, s'est traduite par une "pénétration de l'électricité dans l'énergie" : la part de celle-là dans celle-ci est passée de $4 \%$ en 1925 à $11 \%$ en 1950 , $20 \%$ en $1970,27 \%$ en 1980 (même chiffre pour la France) ; les hypothèse de croissance retenues conduisent à $40 \%$ en 2000 .

L'hydroélectricité s'est accrue, à un rythme moins rapide que celui de l'électricité mais supérieur ou égal à celui de l'énergie. On admet pour l'avenir un taux de croissance toujours intermédiaire entre celui de l'électricité et de l'énergie, soit 3,5\%. Cette croissance intermédiaire a deux conséquences pour l'hydroélectricité :

- La part de celle-ci dans l'électricité s'est réduite passant de $40 \%$ en 1925 à 30\% en 1960, 20\% en 1980 pour arriver à $15 \%$ en 2000 avec les hypothèses de croissance retenues;

- sa part dans l'énergie par contre s'est accrue, passant de $1,7 \%$ en 1925 à $4,4 \%$ en 1950 et $5,3 \%$ en 1974 ; l'accroissement est faible depuis 1950, car fortuitement la production d'hydroélectricité a augmenté sensiblement au même taux que celle de l'énergie ; avec les hypothèses de croissance retenues la part de l'hydroélectricité dans l'énergie devrait augmenter légèrement d'ici l'an 2000 et se situer entre 6 et $7 \%$ (en France, le taux actuel est de $7,3 \%$ ), ce qui ne manque de surprendre quand on perd de vue les hypothèses de croissance retenues, soit $2,5 \%$ pour l'énergie et $3,5 \%$ pour l'hydraulique.

\section{Résumé}

\section{Hypothèses admises}

taux de croissance de l'économie . . . . . . $3 \%$ taux de croissance de la production d'énergie... 2,5\% $" \quad " \quad " \quad$ d'électricité $5 \%$ " d'hydroélectricité ... 3,5\%

Résultats en découlant :

l'hydroélectricité en l'an 2000 compterait pour 3500 TWh $(800 \mathrm{GW})$ et représenterait alors :

- le double de sa capacité actuelle ;

- $35 \%$ du potentiel équipable ;

- $15 \%$ de la production d'électricité ;

- 6,5\% de la production d'énergie.

(14) Dernière année pour laquelle sont publiées en 1981 les statistiques mondiales (World Energy Supplies - ONU New-York).

(15) M. Pecqueur indique en [12] 2700 Mtep d'électricité en 1990, alors qu'au Tableau VIII on a indiqué 3040 Mtep. M. Pecqueur retient donc l'hypothèse d'une croissance de $4 \%$ et non de $5 \%$ de la production totale d'électricité jusqu'en 1990 . Si non, la part du nucléaire serait de $21,4 \%$, comparable aux $21,7 \%$ de l'hydraulique en 1978.

\section{Conclusion}

Elle tient en trois points :

1) En 1978 (14) l'hydroélectricité tient globalement une place modeste dans la production d'énergie $(5,5$ à $6 \%$ ) mais elle représente $22 \%$ de la production d'électricité ; grosso modo, on peut dire qu'elle tient actuellement par rapport à l'énergie et l'électricité, la place que l'on assigne au nucléaire en 1990 [12] : $24 \%$ de la production mondiale d'électricité et $7 \%$ de la production mondiale d'énergie primaire (15).

2) La part équipée du potentiel équipable n'est que de $17 \%$.

3) Sa concentration sur certains pays et certains sites permet de penser que l'équipement hydroélectrique va s'étirer encore sur toute la première moitié du $\mathrm{XXI}^{\mathrm{e}}$ siècle : nous ne serions temporellement parlant, qu'à mi-parcours (1910-1980-2050). De façon imagée, notons qu'il faut marquer en deuxième mi-temps quatre fois plus de buts qu'en première : c'est dire l'ampleur des tâches qui attendent bureaux d'études, entrepreneurs, constructeurs de matériel. ., et financiers.

\section{Références bibliographiques}

[1] L'offre mondiale de charbon. Jacques Petitmengin et M. Ippolito-Charbonnages de France. Pétrole et Technique. Octobre 1981, publiée par l'Association Française des Techniciens du Pétrole, 14, avenue de la Grande Armé 75017 Paris, page 26

[2] World Energy Supplies 1973-1978, ONU, New-York, 1979. Departement of International Economic and Social Affairs. Statistical Office. Statical Papers Series $\mathbf{J}$ No. 22 , page 275

[3] Idem [2], page 2

[4] Enquête sur les Ressources Energétiques. Conférence Mondiale de l'Energie (CME). 1974 publie par The United States Nat. Ctee of the World Energy Conférence, 345 East 47 th Street, New-York, N.Y., 10017.

[5] World Energy Resources 1985-2000 - CME - 1978, publié par IPC Science and Technology Press for WEC, simultanément dans le Royaume-Uni et aux Etats-Unis.

[6] Enquête sur les Ressources Energétiques CME. 1980 préparée par l'Institut Fédéral des Sciences de la Terre et des Ressources Naturelles. Hanovre RFA. Le Tableau 4-7, non cohérent avec celui de la page précédente (349), paraît erroné.

[7] Le Courrier de l'UNESCO. Juillet 1981, publié par IUNESCO, Place de Fontenoy 75700 Paris, pages 9, 12 et 27 .

[8] Statistiques pétrolières \& énergétiques 1980. Dépliant du 18.02.81 édité par le Comité Professionnel du Pétrole 51 , boulevard de Courcelles 75008 Paris.

[9] Mise en service de l'aménagement de Cabora Bassa-Apollo à haute tension continue. Rapport 14-13, CIGRE, Session de 1978 .

[10] Une vue d'ensemble du comportement en service des réseaux de transport à courant continu dans le monde au cours de la période 1975-1978. Rapport 14-08, CIGRE, Session de 1980.

[11] Hydrólectricité de faible puissance. Session de la SHF des 17 et 18 mars 1981 , Houille Blanche $n^{\circ} 4-5,1981$.

[12] L'offre mondiale d'énergie nucléaire, Michel Pecqueur CEA, Pétrole et Technique, octobre 1981, voir [1]. 


\section{Discussion}

Président : M. M. COUPIN

Le Président. - Je remercie M. CoTıLon qui non seulement a strictement respecté l'horaire imparti, mais a fait en outre un exposé extrêmement riche et nourri. . . ce qui, je pense, va également permettre une discussion intéressante entre la salle et lui-même.

S'agissant d'un exposé aussi complet, il est difficile d'en résumer les points forts.

M. COTILLON à juste titre, a insisté sur les incertitudes ; mais je dirai que si l'on prenait d'autres sources d'énergie on aurait certainement à mettre l'accent sur les incertitudes dans le domaine retenu.

On constate en effet dans le domaine de l'énergie une très grande pauvreté en ce qui concerne le recensement des ressources disponibles. Cé qui est sûr, c'est que les diverses ressources en énergie fossile de la planète ne sont pas inépuisables et qu'il convient d'éviter des utilisations excessives du pétrole. Il est donc important, pour un certain nombre de pays, de rechercher l'utilisation optimale des énergies renouvelables sans oublier que l'hydroélectricité est la première d'entre elles aujourd'hui. Cette énergie a d'ailleurs encore un énorme avenir devant elle, comme l'a montré M. CoTilloN, compte tenu de l'écart important entre le potentiel équipé et le potentiel équipable et ceci quelles qu'en soient les incertitudes.

Je passe maintenant la parole à la salle pour les questions.

M. J. de BEAUREGARD. - Le panorama hydroélectrique mondial présenté par M. COTILLON donne l'occasion d'émettre le vœu que je confie aux personnalités du Ministère et de l'EDF qui sont à la tribune : "que la France célèbre avec faste le centenaire de l'électricité" (1).

Les raisons de cette manifestation sont sérieuses :

- la France est le berceau de l'électricité industrielle en général et de l'hydroélectricité en particulier : la houille blanche étant née près de Grenoble, vers 1882 ;

- l'électricité a métamorphosé la vie familiale, agricole, industrielle, les transports, les services et les loisirs. Hier inconnue, aujourd hui indispensable, elle survivra à d'autres sources d'énergie : dans un siècle ou moins, le gaz et le pétrole auront disparu ou presque disparu alors que l'électricité continuera son développement :

- enfin, et surtout pour nous Francais, la crise économique mondiale actuelle est arrivée par le pétrole et la France ne pourra sortir de cette crise qu'en utilisant au maximum le vecteur énergétique qu'est l'électricité d'origine nucléaire et hydraulique.

Pour toutes ces raisons, la SHF devrait inciter les autorités compétentes à organiser le centenaire de l'électricité.

M. le Président. - Je dirai que l'objectif premier de la politique des Pouvoirs Publics dans le domaine de l'énergie est justement d'échapper au pétrole. C'est d'ailleurs ce que le large débat public de l'automne dernier a montré, avec le plan énergétique approuvé par le Parlement, plan qui laisse une très large part à l'électricité.

Bien sûr, cette recherche de l'indépendance énergétique ne concerne pas seulement l'électricité : l'électricité est un moyen et non pas une fin : et l'on passe d'une énergie dominante à un ensemble d'énergies diverses. La caractéristique de l'ère dans laquelle nous sommes entrés, c'est qu'il ne doit plus y avoir d'énergie dominante : on doit utiliser les énergies les plus variées de manière à échapper le plus vite possible à la dépendance pétrolière.
Cette place importante, mais non exclusive, de l'électricité étant rappelée, nous étudierons avec intérêt la suggestion de M. de BEAUREGARD.

M. GoUbET. - Dans son exposé, M. COTIllon conformément à la pratique usuelle, nous a présenté l'évolution de l'hydroélectricité en parlant essentiellement de $\mathrm{kWh}$. Or, on constate que plusieurs pays industriels (France, Italie, Suisse, Suède...) quí ont à peu près terminè la mobilisation de leur potentiel hydroélectrique n'en continuent pas moins à déployer une grande activité dans ce domaine (indépendamment des usines de pompage) en suréquipant les usines existantes ; ces travaux n'augmentent pratiquement pas la production mais permettent de mieux placer, au moment des pointes de consommation, la production hydroélectrique qui représente un pourcentage relatif de plus en plus taible mais instantané de la consommation électrique totale. La notion de potentiel équipable (ou de production) exprimée en kWh ne permet pas de rendre compte de cet aspect du problème. Ne pourrait-on pas le prendre en compte, par exemple, en ajoutant à la notion de production annuelle celle d'énergie susceptible d'être stockée dans les réservoirs.

M. COTILloN. - Vous éclairez là un point particulier du panorama et c'est une autre façon de mettre en lumière le rôle que va continuer à jouer l'hydraulique sous la forme de suréquipements. Votre remarque comme toujours est excellente, mais dans les publications et les statistiques actuellement disponibles on en traite fort peu. Je n'ai donc pas eu matière à en débattre.

M. le Président. - Dans la très grande diversité des pays décrits par M. COTILLON toutes sortes de situations diverses d'utilisation de l'hydroélectricité se présentent : dans certains pays c'est l'apport en énergie qui est fondamental, dans d'autres comme en France, où le système énergétique est plus évolué et plus complexe, c'est la capacité de stockage d'électricité potentielle que permet l'hydroélectricité qui prend une importance croissante. La remarque de M. GOUBET peut certainement se situer dans ce cadre. C'est une raison de plus pour espérer que l'hydroélectricité ait encore un long avenir devant elle.

M. de MAUBLANC. - Pour compléter la remarque de M. GOUBET, je dirai que le sujet qui le préoccupe est précisément celui qui a retenu l'attention de certaines organisations internationales. Le problème que vous avez évoqué, qui se pose en France, en Suède, etc. . a aux statisticiens sur la façon de prendre en compte cette énergie stockée, fait l'objet d'études dans le cadre de I'UNIPEDE; nous aurons l'occasion d'en reparler peut-être dans une autre enceinte; mais je peux vous dire que c'est un problème au cour des préoccupations actuelles.

M. Le Président. - Pour rassurer M. GOUBET sur le peu de place que tient le suréquipement dans notre réunion de ce matin, je lui rappelle que M. DAUZIER présentera cet aprèsmidi une communication précisément sur ce sujet.

D'autres interventions ?... Non ... Je crois que ce silence traduit le caractère très complet de votre exposé et l'accord des participants sur vos conclusions et sur la place que vous avez faite au caractère quasi prométhéen de cette énergie tout à tait particulière et importante.

Je vous propose de passer au deuxième exposé de la matinée, celui que vous fera $M$. de MAUBLANC.

(1) Cet anniversaire a été célébré le 15 juin 1982 au Conservatoire National des Arts ct Métiers par le professeur A. BUSSON, titulaire de la chaire occupée naguère par Marcel DEPREZ (1843-1918). 
M. H. de MAUBLANC. - Tout à l'heure un intervenant a évoqué la perspective de la célébration du centenaire de l'électricité. A supposer qu'on puisse fixer une origine quelconque à l'électricité ou à son usage industriel (une origine en vaut une autre), pour ma part je préfère me référer aux années 1885 où les premières liaisons électriques de quelque importance en puissance et en distance ont été créées dans les Alpes. C'est dire que dans notre pays (dont certains s'accordent à penser qu'il est l'inventeur de l'électricité industrielle et domestique) nous avons une centaine d'années d'expérience, ce qui est considérable.

Mais je dois dire que le sujet qui m'a été proposé m'a laissé quelque peu perplexe, puisqu'il s'intitule : les développements récents en France de l'ingéniérie en hydroélectricité. . .

\section{Abstract \\ Share of hydro-electric power in the world energy balance}

Hydro power and other renewable energy sources arise from features that cannot be dissociated from the place they occur, unlike solid, liquid or gazeous fossil fuels of nonrenewable energy sources that are readily transportable. Hydro power is only able to come into its own in combination with electricity, but river harnessing has always been seen as man's triumph over the forces of nature and has become something of a religious cruisade.

Total developabre hydro potential is estimated at 10000 $12000 \mathrm{TWh}$, repressenting $27 \%$ to $33 \%$ of the total theoretical potential put at $36000 \mathrm{TWh}$. But authors calculate in energy or capacity using different units of measurement, and there are many errors in the literature. Hydro power is more evenly distributed throughout the world than coal, oil, gaz or uranium reserves although within large regions like the African continent, there is considerable imbalance with most of the potential being concentrated in a few countries or even at a few sites like Inga.

In 1978 developed potential (expressed as actual electricity output in that year) represented $17 \%$ only of developable potential $(98000 \mathrm{TWh}$ ) and $21.7 \%$ of total electricity production. But two-thirds of the developed potential was confined to the 24 OECD countries, which have harnessed $50 \%$ of their potential on average; France, by the end of 1981, had reached a figure of $90 \%$.

By the year $2000,35 \%$ might be developed, with the OECD countries accounting for $15 \%$, USSR, India, Mexico, Brazil and Yugoslavie $12 \%$, and the rest of the world $8 \%$.
Long transmission distances will not be a brake on development. The new thyristor rectifier technology for large-capacity d.c. lines is promising.

Mini-hydro is not a universal cure and can only contribute a few percent of output.

Pumped storage, expanding fast in Japan, USA and Western Europe is just as attractive to engineers and the construction industry as conventional hydro but is does not produce energy ; it merely stores it for later use. The plants are sometimes very large indeed (1 $800 \mathrm{MW}$ at Dinorwic in Wales).

Conventional hydro schemes are also growing in size, being increasingly sited on large rivers in the plains $\left(10000 \mathrm{~m}^{3} / \mathrm{s}\right.$ average river flow at Itaipu in Brazil). Where alternative capacity exists on the grid, hydro is being used for peaking duty (10000 MW at Grand Coulee on the Columbia and at Guri on the Caroni in Venezuela).

The share of hydro in total electricity will have dropped from $40 \%$ in 1925 to $13 \%$ by the year 2000 . But its share in total energy, which rise from $1.7 \%$ in 1925 to $5.3 \%$ in 1974 , is expected to reach 6-7\% by the end of the century since predicted annual growth rates are : total energy $2.3 \%$, hydro $3.5 \%$, total electricity $5 \%$, for a world econrmic growth of $3 \%$.

By 1978 , hydro power occupied the same place in electricity output as nuclear power is expected to do by 1990 . 